\title{
A Review of Personal Communications Services
}

\author{
Hui Cheng ${ }^{1}$, Xingwei Wang ${ }^{2}$, Min Huang ${ }^{2}$, Shengxiang Yang ${ }^{1}$ \\ ${ }^{1}$ Dept of Computer Science, University of Leicester, University Road, Leicester LE1 7RH, UK \\ ${ }^{2}$ College of Information Science and Engineering, Northeastern University, Shenyang, China \\ \{hc118,sy11\}@mcs.le.ac.uk, \{wangxw,mhuang\}@mail.neu.edu.cn
}

\begin{abstract}
PCS is an acronym for Personal Communications Service. Ubiquitous PCS can be implemented by integrating the wireless and wireline systems on the basis of intelligent network (IN), which provides network functions of terminal and personal mobility. In this chapter, we focus on various aspects of PCS. First we describe the motivation and technological evolution for personal communications. Then we introduce three key issues related to PCS: spectrum allocation, mobility, and standardization efforts. Since PCS involves several different communication technologies, we introduce its heterogeneous and distributed system architecture. Finally, IN is described in detail because it plays a critical role in the development of PCS.
\end{abstract}

Keywords: Personal communications services, personal communication networks, intelligent network.

\section{Introduction}

Personal communications services (PCS) has two layers of meaning. At the low layer, from the technical perspective, PCS is a $2 \mathrm{G}$ mobile communication technology operating at the $1900 \mathrm{MHz}$ frequency range. At the upper layer, PCS is often used as an umbrella term that includes various wireless access and personal mobility services with the ultimate goal of enabling users to freely communicate with anyone at anytime and anywhere according to their demand.

In daily life, the vast majority of us will communicate with each other, no matter where we are, through various handheld communication devices. With the social development, people have proposed more and more personalized communication demands. Scientists and engineers need to develop more and more powerful communication technologies to meet the ever-increasing demands. There is no universally accepted definition for PCS. PCS is a vague term that expresses the service capabilities of all types of personal communications [1]. Personal communication services provide personalized voice, data, image, and video communications services anywhere, anytime, with anybody. PCS makes communication independent not only of distance and location, but also of time. The realization of personal communications services makes some requirements necessary on various types of networks, in particular, mobility and interworking.

From the technical perspective, PCS is the U.S. Federal Communications Commission (FCC) term used to describe a set of digital cellular technologies deployed in the U.S. Also referred to as digital cellular, PCS works over CDMA (also called IS-95), GSM, and North American TDMA (also called IS-136) air interfaces. PCS still belongs to $2 \mathrm{G}$ mobile communications technology and includes enhanced personal communications services such as SMS text messaging or caller ID. However, PCS is not a totally new $2 \mathrm{G}$ technology. Three of the most important features of PCS systems are: 1) completely digital; 2) operating at the $1900 \mathrm{MHz}$ frequency range (unlike other cellular systems that operate in the $800 \mathrm{MHz}$ frequency range); 3) they can be used internationally.

\section{Technological evolution for personal communications}

To understand PCS, we first review the history of technological development in personal communications.

Mobile Radio: Mobile radio is the earliest system for personal communications. It was proprietary and costly. It could be used within a certain region.

Cellular Telephone: It was not until 1983 that today's analog cellular services were introduced to the U.S. on a commercial basis. However, it is the crucial step towards PCS.

Cordless telephone: It is a telephone with a wireless handset which communicates with a base station connected to a fixed telephone landline via radio 
waves. It can only be operated close to (typically less than 100 metres of) its base station.

Cellular Data: $2 \mathrm{G}$ is based on circuit-switched technology where each call requires its own cell channel, which makes transmission of data quite slow. The representative technology of $2 \mathrm{G}$ is GSM (Global System for Mobile communications).

Satellite Communications: True personal communication must be ubiquitous, meaning that twoway voice and data should be available anywhere in the world, no matter how remote or underdeveloped that location [2].

$P C N$ : Expansion from cellular to personal communications services occurred at a quickening pace worldwide. Personal communications networks (PCN's) provide mobile two-way and mass-market communication services, the most advanced offering of the PCS area.

Internetwork: An Internetwork functions as a single large network, which is a collection of individual networks connected by intermediate networking devices.

\section{Key issues in PCS}

\subsection{Spectrum allocation}

The 1992 World Administrative Radio Conference (WARC92) of the ITU resulted in a worldwide allocation for mobile services in the 1.7 to $2.69 \mathrm{GHz}$ band [3]. This brought all three regions of the world into conformity under the Future Public Land Mobile Telecommunications Systems (FPLMTS, now IMT2000), which are systems capable of providing a wide range of services including personal communications with regional or international roaming. The FPLMTS concept incorporates both terrestrial and satellite-delivered PCS services. The conference identified the sub-bands 1885 to $2025 \mathrm{MHz}$ and 2110 to $2200 \mathrm{MHz}$ for implementation of terrestrial PCS components on a worldwide basis. The conference also allocated spectrum for LEO satellite services that can provide PCS-type services to remote areas. For LEO systems operating below $1 \mathrm{GHz}$, a primary global allocation in the 149.9 to $150.05 \mathrm{MHz}$ band was made and secondary allocations at 312 to 315 $\mathrm{MHz}$ and 387 to $390 \mathrm{MHz}$ were made. For LEO systems above $1 \mathrm{GHz}$, primary allocation in the 1610 to $1625.5 \mathrm{MHz}$ band (Earth to space) paired with the 2483.5 to $2520 \mathrm{MHz}$ band (space to Earth) and a secondary allocation at 1613.8 to $1626.5 \mathrm{MHz}$ were made.

The spectrum allocated to PCS is divided into three major categories: broadband, narrowband, and unlicensed. The FCC controlled the spectrum allocation for broadband PCS in the U.S., and defined rules for auctioning the spectrum to potential PCS service providers. The most challenging and controversial decision confronting the FCC was to determine a frequency allocation for PCS that takes into account the competing demands of existing licenses for scarce spectrum. Fortunately, these new rulings received a lot support and praise by the industry [4]. Broadband PCS operates in the 1850-1910 MHz and 1930-1990 MHz bands. Narrowband PCS operates in the 901-902 MHz, 930-931 MHz, and 940$941 \mathrm{MHz}$ bands. For unlicensed PCS services, a 20 $\mathrm{MHz}$ spectrum in 1910-1930 MHz band was allocated.

Since the spectrum resource is not unlimited, one of the most fundamental issues concerns overcrowding on the radio frequency spectrum. Efficiently sharing the spectrum resource is of paramount importance in wireless communication systems, in particular in personal communications where large numbers of wireless subscribers need to be served [5]. There is always a desire to promote increased flexibility, innovation, and efficient usage of the spectrum resource. The frequencies most favored are below 1 $\mathrm{GHz}$ because they diffract around objects better, but this limited bandwidth could not satisfy the demand without reusing the band many times [6]. The simplest form of reuse is geographic separation. In addition, one of the most promising alternatives to spectrum reallocation is to use the current spectrum more efficiently. By using multiple access, digital technology improves frequency efficiency and decreases costs to the point that personal communications appeal to people around the world. Multiple access refers to the simultaneous transmission by numerous users to or through a common receiving point. The three types of multiple access presently used with personal communications are code-division, frequency-division, and time-division multiple access, i.e., CDMA, FDMA, and TDMA.

\subsection{Mobility}

PCS supports the mobility of users both as they roam from system to system between phone calls, and supports handoffs between base stations within and between adjacent systems [7]. The functions of mobility management include: handoff, location management, and registration for service while roaming. The functions control the location of PCS end users, the use of terminal equipment, and the availability of PCS anytime, anywhere. Three types of mobility have to be considered in PCS [1]: terminal mobility, personal mobility, and service mobility (also 
called service portability). According to the definition given by the T1P1 Committee of the American Telecommunications Standards Institute (ATSI), PCS must contain the following three functionalities:

1) Terminal Mobility From the user's viewpoint, it is the ability of a mobile and wireless terminal to access communication services from different locations while in motion. From the service provider's viewpoint, it is the ability of the network to identify, locate, and track the mobile terminals.

2) Personal Mobility Personal mobility also has two layers of meaning. From the user's viewpoint, it is the ability of a user to access communication services based on a personal identification code on any terminal irrespective of wireless or wireline connection. From the service provider's viewpoint, it is the ability of the network to locate the terminal associated with the user and provide those services according to the user's service profile. Once the terminal associated with one user is located, the network needs to do addressing, routing, and charging about the user's calls.

3) Service Profile Management It refers to the ability of a user to access and manipulate the user's service profile. Service profile management may be realized by the database system, which controls the access to and handling of data. The consistency of information in the service profile can be guaranteed by the service profile management functions.

4) Service Mobility It can be seen as a combination of terminal mobility and personal mobility, is related to service profile management. Service mobility refers to the network capability to provide subscribed services (e.g., a user's individual service profile at a userdesignated terminal or location) and identify the user at any access location by supporting terminal and/or personal mobility.

\subsection{Standardization efforts}

PCS is based on digital architecture, generally using TDMA or CDMA protocols. A remarkable technical characteristic of PCS is its high capacity and spectral efficiency. International standards were developed to meet the global standardization demand for PCS equipments and systems. For specifying the functionalities and standards of PCS, the standardization organizations include ITU, ETSI (European Telecommunications Standards Institute), TIA (Telecommunications Industry Association, affiliated with the Electronic Industries Association), and the Committee T1. A joint technical committee between T1P1 and TR46 subcommittees has coordinated the joint work on the PCS standards between $\mathrm{T} 1$ and TIA. The joint technical committee has defined methodology and selection criteria in selecting appropriate radio interface technology.

In U.S., the T1P1.2 and T1P1.3 have defined highlevel requirements for PCS standardization and documented these in technical reports. The T1P1.2 developed a reference model for PCS and identified six different configurations as potential ways of implementing the reference model [8]. T1P1 also has played a significant role in defining personal mobility, also known as UPT (Universal Personal Telecommunications). TR-46 subcommittee of TIA specified a reference model from the perspective of a cellular service provider [9]. Other organizations such as PCIA (Personal Communications Industry Association) also have specified PCS reference models and proposed implementation options [10]. PCS data standards were investigated by the joint technical committee of Committee T1 and the TIA, the ITU-T (ITU-Telecommunications standardization sector).

\section{System architecture}

\subsection{Heterogeneous PCS (HPCS)}

PCS may appear in many forms and consist of a plethora of systems that address cordless phone, cellular, vehicular, and a variety of other services. Two of the most popular technologies used in PCS are cellular telephony and cordless telephony. Cellular systems can be AMPS, GSM, ADC (American Digital Cellular or IS-54, and the newer version, IS-136), and DCS1800 (Digital Communication System at 1800 $\mathrm{MHz}$, et al. Among cordless telecommunication systems exist the CT2 (Cordless Telephone 2), DECT, PACS (Personal Access Communications Systems), and PHS. Integration of different PCS systems was proposed, particularly integration of cellular and cordless systems to provide better services than any individual system. The integration of these different systems is referred to as "heterogeneous PCS (HPCS) " [11].

In HPCS, the service areas of different individual PCS systems may overlap or not. If there is an overlapping, the system typically operates at different frequency bands, and the integration can increase the system capacity. If there is no overlapping, obviously the integration of these systems can extend the coverage of the service area. For example, a simple HPCS is formed by integrating a cellular system and a cordless telecommunication system. In such a simple HPCS, from the perspective of the cellular system, the quality of circuits is improved; and from the perspective of the cordless system, higher user mobility is supported. 
A basic requirement of HPCS is downward compatibility. In HPCS, users will receive services from multiple PCS systems since hey have been integrated. At the same time, the original users of the individual PCS systems will still receive services from their systems without being affected by HPCS. Depending on the network and radio technologies, in [11], three types of integration are considered: SRSN (Similar Radio technology, Same Network technology), DRSN (Different Radio technology, Same Network technology), DRDN (Different Radio technology, Different Network technology). Different integration type has significantly different effect on the implementation of HPCS. One obvious issue is handover between different systems. Another important issue is roaming management which involves three aspects (registration, call delivery, and handset identity). Both issues were deeply investigated in implementations of HPCS.

\subsection{Distributed network architecture}

Centralized cellular network architectures are likely to be unsuitable for management of the anticipated PCS demand, including the associated signaling arising. So, distributed network architecture to support PCS demand should be designed to incur low network signaling overhead. In [12], a distributed microcellular architecture based on the IEEE 802.6 metropolitan area network (MAN) was proposed to facilitate PCS deployment. The MAN supports both voice and data traffic using a dual bus architecture. The Mobile Control Center (MCC) on the MAN has the responsibility of managing the mobile aspects of calls. The Signaling Terminal (ST) on the MAN is to manage the fixed parts of all calls. Fixed and wireless terminals connected to LANs and PBXs provide PCS coverage within buildings. To provide a contiguous coverage in public areas, sets of base stations controlling microcells are interconnected to MANs via heterogeneous bridges. A MAN-oriented microcellular system architecture can reduce traffic congestion by dynamic sharing of data link capacity and by partitioning call control functions to a distributed switch.

In [13], another distributed architecture called Wireless Distributed Call Processing Architecture (WDCPA) was proposed. In WDCPA, a new network element named the user signaling server is added, which provides proxy services on behalf of mobile users and helps increase signaling efficiency. In addition, with the advent of multimedia services and multiconnection mobile computing applications (e.g., Internet multimedia multicast), multi-connection calls become more and more important. To support multiconnection services, WDCPA separates connection control from call control in its architecture. WDCPA also distributes call processing functions from switching entities. Thus, more efficient control procedures are allowed to be developed since the processing for a control function can be placed closer to the data needed to perform the function. In WDCPA, the wired network infrastructure is assumed to be BISDN standards in order to support multimedia and other QoS-dependent services. However, the mobility management algorithms and structure of WDCPA do not depend on the wired infrastructure.

\subsection{Intelligent network}

Intelligent network (IN) is an architectural concept of telecommunication networks, which is formally defined by ITU-T in 1992. It provides a flexible and open architecture to improve service implementation and provisioning in a more cost-effective manner than traditional concepts [1]. To provide a ubiquitous PCS, it is essential to integrate different telecommunication networks and systems. In fixed networks, intelligent network has been introduced to facilitate the development of new services. IN was also used to integrate mobility in fixed networks. In mobile cellular networks, although mobility management has already existed as a component, it is desirable to introduce the IN concept to increase the flexibility of such networks, allowing an improved services provision. With the convergence of fixed networks and mobile networks becoming more and more popular, the IN concept can be utilized to provide necessary networking functions for their integration.

There are two types of INs in the world. One has been developed by ITU and called IN Capacity Set-x (CS-x). This is an international IN standard. The other is the Advanced Intelligent Network (AIN), which has been standardized by Bellcore in the U.S. We focus on the international IN standard. ITU-T has realized the Recommendations concerning IN by three phases: Capacity Set-1 (CS-1), Capacity Set-2, Capacity Set-3. CS-1, the first phase of IN systems, defines a complete architecture including the architectural view, state machines, physical implementation and protocols. It is the formal indication that the IN was produced. CS- 2 includes many new capabilities over CS-1. It has addressed key issues such as B-ISDN, mobility, and multimedia services. CS-2 also makes standards to cover the service creation, service management functions. CS-3 is a superset of CS-2, thus all parameters supported in CS-2 are also supported in CS-3. CS-3 supports IMT-2000 for the realization of 
mobile multimedia service, and provides the standards to combine IN, B-ISDN, and Telecommunications Management Network (TMN). Summarizing, we can relate broadly the CS- 1 to PSTN and ISDN, the CS-2 to B-ISDN, and the CS-3 to the fixed and mobile networks.

The IN Conceptual Model (INCM) specifies the framework to describe and design IN architectures. The INCM provides four views of a telecommunication network through which a given service is modelled. These views correspond to four planes [14]. In the following, we describe them one by one.

The service plane (SP) presents to end users an abstract view of the services provided by an IN-based network. In this plane, an IN service is described by a set of service features (SF's).

The global functional plane (GFP) provides a view to design services. SFs are realized by components called service-independent building blocks (SIB's). Thus, an IN service is built by a set of SIBs which represents the service logic (SL) used to realize the service.

The distributed functional plane (DFP) defines functional entities (FE's) to characterize the functional aspect of the network. These encompass actions called functional entity actions (FEA's) invoked by the SIBs. The IN FEs include call control agent function (CCAF), service creation environment function (SCEF), service management access function (SMAF), service switching function/call control function (SSF/CCF), service management function (SMF), service control function (SCF), service data function (SDF), and specialized resource function (SRF). IN Application Protocol (INAP) is the protocol used by the FE to communicate with each other. Three categories of functions are identified in the DFP: the access functions -invoked during the access of a given service and for the connection between users; the intelligence functions - used to perform a given service; and the management functions -dedicated to the service creation and service management.

The physical plane (PP) shows how the different FE of the DFP is mapped onto physical entities (PE). Physical entities communicate with each other through a communication protocol. The IN PE include service switching point (SSP), service control point (SCP), service management point (SMP), and intelligent peripheral (IP). Among them, SSP, SCP, and IP are in conjunction responsible for the real-time execution and control of the end-user services. The SSP capabilities are defined in terms of trigger check points (TCP's), and the SCP and IP capabilities in terms of FEAs.

The application of IN to fixed and mobile networks is very convenient to realize PCS. PCS was also regarded as a leading driver for deployment of intelligent network. Modelling PCS in IN means that services are specified with entities associated to INCM in each of the four planes. In the SP, PCS is defined with pre-existent SFs, which are usable to specify several services. In the GFP, PCS is built with the description of a sequence of SIB to generate their SL. In the DFP, PCS is decomposed into FE distributed in an IN environment. In the PP, PE contain the FE of DFP.

In [15], it proposed to outline the IN requirements for implementing PCS in the following steps.

1) Identify a set of PCS feature requirements needed by end users.

2) Identify the specific TCPs and FEAs required to implement the PCS features.

3) The final result is a subset of IN capabilities that can be rationalized against a specific service. As such, these capabilities could be used as the starting point for an economic analysis of IN implementation cost versus service worth.

The industry also used IN to deploy PCS in order to support interworking and interoperability. PCS wireless system needs to accommodate mobility of all its intended users. To support the user mobility, the cellular and PCS systems need to interoperate to maintain a call. The different types of wireless systems also utilize the capabilities of the IN to provide seamless roaming [16]. Interoperability between service providers will minimize the user interface inconsistencies that may be caused by inherently different system implementations. For example, MSCbased systems utilize IN elements (HLR and VLR) along with a mobile switching center (MSC) to provide high-mobility PCS capability where service providers wish to operate and manage their own systems. In Class 5 switch-based systems, use of standard wireline interfaces and capabilities allows various PCS network elements to coexist. This architecture incorporates major IN elements, and provides the evolutionary path to migrate the PCS system to both low- and highmobility PCS traffic [17].

The IN intersystem protocols are IS-41 MAP (defined by EIA/TIA IS-41C, MAP stands for mobile application part), PCS 1900 MAP (PCS 1900 is a derivative of GSM), and advanced intelligent network (AIN). Interworking and interoperability between IS41 MAP and PCS 1900 MAP systems is a key issue in ensuring that seamless roaming is provided to mobile users. These intersystem protocols play a key role in interconnecting various network elements to the MSC or SCP to a Class 5 switch. By these protocols, diverse networks are interconnected to offer ubiquitous service, universal accessibility, and seamless roaming. 


\section{Conclusion}

PCS is a set of capabilities that allows some combination of terminal mobility, personal mobility, and service profile management. That is, it emphasizes person-to-person rather than traditional point-to-point communication. The services provided by PCS are more convenient, ubiquitous, and versatile than the public has ever received. Technically, cellular systems in the U.S. operate in the $824-849 \mathrm{MHz}$ frequency bands; PCS operates in the $1850-1990 \mathrm{MHz}$ bands. A typical PCS network would use uniform microcells with a high degree of frequency reuse.

PCS has two outstanding characteristics: support mobile users, and support multimedia communication. The "personal" in PCS also distinguishes this service from cellular by emphasizing that, PCS is designed for greater user mobility. It generally requires more cell transmitters for coverage, but has the advantage of fewer blind spots. Ubiquitous PCS can be implemented only by integrating the wireless and wireline systems on the basis of intelligent network. Furthermore, advanced PCS system architectures must account for the increasing demand for Internet access and other private data service requirements.

\section{Acknowledgment}

This work was supported by the National HighTech Research and Development Plan of China under Grant No. 2006AA01Z214; the National Natural Science Foundation of China under Grant No. 60673159 and No. 70671020; Program for New Century Excellent Talents in University; the Key Project of Chinese Ministry of Education under Grant No. 108040; Specialized Research Fund for the Doctoral Program of Higher Education under Grant No. 20060145012 and No. 20070145017; the Natural Science Foundation of Liaoning Province under Grant No. 20062022, and the Engineering and Physical Sciences Research Council (EPSRC) of UK under Grant EP/E060722/1.

\section{References}

[1] Nadege, F. and Cegetel, T.H. Personal communications services through the evolution of fixed and mobile communications and the intelligent network concept. IEEE Network, Vol. 12, No. 4, pp. 11-18, 1998.

[2] Viterbi A.J. The evolution of digital wireless technology from space exploration to personal communication services. IEEE Transactions on Vehicular Technology, Vol. 43, No. 3, pp. 638-644, 1994.

[3] Wimmer, K.A. and Jones, J.B. Global development of PCS. IEEE Communications Magazine, Vol. 30, No. 6, pp. 22-27, 1992.

[4] Hemmady, J.G., Maymir, J.R., and Meyers, D.J. Network evolution to support personal communications services. Proc. IEEE GlobeCom, 1994.

[5] Rosberg, Z. and Zander, J. Toward a framework for power control in cellular systems. Wireless Networks, Vol. 4, No. 4, pp. 215-222, 1998.

[6] Papatriantafilou, M., Rutter, D., and Tsigas, P. Distributed frequency allocation algorithms for cellular networks: trade-offs and tuning strategies. Proc. 13th IASTED PDCS, 2001.

[7] Stuart, J.L. Personal communications networks bridging the gap between cellular and cordless phones. Proceedings of the IEEE, Vol. 82, No. 4, pp. 564-571, 1994.

[8] A technical report on network capabilities, architectures, and interfaces for personal communications. Tech. Rep. T1Rpt 34-1994, 1994.

[9] Personal communications service descriptions. Tech. Rep. TR46 - Draft PN-3168, 1993.

[10] PCS standards requirements document - service description standard. Tech. Rep. TE/92-07-09/104, Telocator (now PCIA), 1992.

[11] Lin, Y.B. and Chlamtac, I. Heterogeneous personal communications services: integration of PCS systems. IEEE Communications Magazine, Vol. 34, No. 9, pp. 106-112, 1996.

[12] Malyan, A.D., Ng, L.J., Leung, C.M.V., and Donaldson, W.R. Network architecture and signaling for wireless personal communications. IEEE Journal on Selected Areas in Communications, Vol. 11, No. 6, pp. 830-841, 1993.

[13] Porta, F.L.T., Veeraraghavan, M., and Buskens, W.R. Comparison of signaling loads for PCS systems. IEEE/ACM Transactions on Networking, Vol. 4, No. 6, pp. 840-856, 1996.

[14] Nadege, F. Intelligent networks: a key to provide personal communications services. Proc. GlobeCom, 1998.

[15] Homa, J. and Harris, S.. Intelligent network requirements for personal communications services. IEEE Communications Magazine, Vol. 30, No. 2, pp. 70-76, 1992.

[16] Gregory, S.L. IN architectures for implementing universal personal telecommunications. IEEE Network, Vol. 8, No. 2, pp. 6-16, 1994.

[17] Husain, S.S. and Marocchi, A.J. Intelligent network: a key platform for PCS interworking and interoperability. IEEE Communications Magazine, Vol. 34, No. 9, pp. 98-105, 1996. 